TRANSACTIONS OF THE

AMERICAN MATHEMATICAL SOCIETY

Volume 364, Number 3, March 2012, Pages 1413-1426

S 0002-9947(2011)05457-7

Article electronically published on October 11, 2011

\title{
UNIQUENESS AND REGULARITY FOR A SYSTEM OF INTERACTING BESSEL PROCESSES VIA THE MUCKENHOUPT CONDITION
}

\author{
SEBASTIAN ANDRES AND MAX-K. VON RENESSE
}

\begin{abstract}
We study the regularity of a diffusion on a simplex with singular drift and reflecting boundary condition which describes a finite system of particles on an interval with Coulomb interaction and reflection between nearest neighbors.

As our main result we establish the strong Feller property for the process in both cases of repulsion and attraction. In particular, the system can be started from any initial state, including multiple point configurations. Moreover, we show that the process is a Euclidean semi-martingale if and only if the interaction is repulsive. Hence, contrary to classical results about reflecting Brownian motion in smooth domains, in the attractive regime a construction via a system of Skorokhod SDEs is impossible. Finally, we establish exponential heat kernel gradient estimates in the repulsive regime.

The main proof for the attractive case is based on potential theory in Sobolev spaces with Muckenhoupt weights.
\end{abstract}

\section{INTRODUCTION AND MAIN RESULTS}

In this paper we consider the regularity of a reversible particle system with singular nearest neighbour interaction, confined to stay in a Weyl chamber by reflection. Brownian motion or more general diffusions in Euclidean domains with reflecting boundary condition are a classical subject in stochastic analysis, with strong connections to boundary regularity theory for parabolic PDE. Starting from the early works by e.g. Fukushima [14 and Tanaka 24, the field has seen perpetual research activity; cf. [6, 18, (cf. also [4] for more references). Likewise, reversible singular particle systems have been studied for some time (cf. [13, 19, 9]), with new interest emerging from random matrix theory (cf. [21, 16]). Systems with both singular drift and reflection at the boundary were studied more recently in [26] and [12].

Apart from being not of mean field but of nearest neighbor type, the process proposed in this work differs from all aforementioned models, in particular from the well studied Dyson-type Brownian motion, in that the interaction between the particles in the interesting regime is not repulsive but attractive. As a consequence, familiar regularity conditions for the reference measure $q(d x)$ such as log-concavity, $q \in H^{1,1}(\Omega, d x)$ in [26] or $\nabla(\log q) \in L^{p}(\Omega, d q), p>N$ in [12] do not hold. Therefore we find new arguments based on harmonic analysis in Sobolev spaces with

Received by the editors November 20, 2009 and, in revised form, May 21, 2010.

2010 Mathematics Subject Classification. Primary 60J60, 42B37.

Key words and phrases. Bessel process, reflecting boundary condition, Coulomb interaction, Feller property, Muckenhoupt weights.

(C)2011 American Mathematical Society Reverts to public domain 28 years from publication 
Muckenhoupt weights. In particular, together with reversibility this yields a short proof for weak uniqueness.

To specifiy our model, let

$$
\Sigma_{N}=\left\{x=\left(x^{1}, \ldots, x^{N}\right) \in \mathbb{R}^{N} \mid x^{0}:=0<x^{1}<x^{2}<\ldots<x^{N}<1=: x^{N+1}\right\}
$$

be the $N$-dimensional Weyl chamber equipped with the measure

$$
q_{N}(d x)=\frac{1}{Z_{\beta}} \prod_{i=0}^{N}\left(x^{i+1}-x^{i}\right)^{\frac{\beta}{N+1}-1} d x^{1} d x^{2} \ldots d x^{N},
$$

where $\beta>0$ is a free parameter and $Z_{\beta}=\left(\Gamma(\beta) /(\Gamma(\beta /(N+1)))^{N+1}\right)^{-1}$ is a normalization constant. We study the process $\left(X^{x}\right)$ induced by the $L^{2}\left(\Sigma_{N}, q_{N}\right)$ closure of the (pre-)Dirichlet form

$$
\mathcal{E}^{N}(f, f)=\int_{\Sigma_{N}}|\nabla f|^{2}(x) q_{N}(d x), \quad f \in C^{\infty}\left(\bar{\Sigma}_{N}\right),
$$

whose generator $\mathcal{L}$ extends the operator $\left(L^{N}, D\left(L^{N}\right)\right)$,

$L^{N} f(x)=\left(\frac{\beta}{N+1}-1\right) \sum_{i=1}^{N}\left(\frac{1}{x^{i}-x^{i-1}}-\frac{1}{x^{i+1}-x^{i}}\right) \frac{\partial}{\partial x^{i}} f(x)+\Delta f(x) \quad$ for $x \in \Sigma_{N}$,

with the domain

$D\left(L^{N}\right)=C_{N e u}^{2}=\left\{f \in C^{2}\left(\bar{\Sigma}_{N}\right) \mid \nabla f \cdot \nu=0\right.$ on all $(n-1)$-dimensional faces of $\left.\partial \Sigma_{N}\right\}$,

and $\nu$ denotes the outward normal field on $\partial \Sigma_{N}$. On the level of formal Itô calculus $\left(L^{N}, D\left(L^{N}\right)\right)$ corresponds to an order preserving dynamic for the locations of $N$ particles in the unit interval solving the system of coupled Skorokhod SDEs

$d x_{t}^{i}=\left(\frac{\beta}{N+1}-1\right)\left(\frac{1}{x_{t}^{i}-x_{t}^{i-1}}-\frac{1}{x_{t}^{i+1}-x_{t}^{i}}\right) d t+\sqrt{2} d w_{t}^{i}+d l_{t}^{i-1}-d l_{t}^{i}, \quad i=1, \ldots, N$,

where $\left\{w_{i}\right\}$ are independent real Brownian motions and $\left\{l^{i}\right\}$ are the collision local times, i.e.

$$
d l_{t}^{i} \geq 0, \quad l_{t}^{i}=\int_{0}^{t} \mathbb{1}_{\left\{x_{s}^{i}=x_{s}^{i+1}\right\}} d l_{s}^{i} .
$$

The process $X=\left(x^{1}, \ldots, x^{N}\right)$ may thus be considered as a system of coupled (two sided) real Bessel processes with uniform Bessel dimension $\delta=\beta /(N+1)$. Similar to the standard real Bessel process $B E S(\delta)$ with Bessel dimension $\delta<1$, the existence of $X$, even with initial condition $X_{0}=x \in \Sigma_{N}$, is not trivial, nor are its regularity properties.

The initial motivation for studying this model was its relation to the Wasserstein diffusion introduced in [28. In [3] we show that the normalized empirical measure of the system (1.3) converges to the Wasserstein diffusion in the high density regime for $N \rightarrow \infty$, assuming Markov uniqueness of the infinite-dimensional Wasserstein Dirichlet form. The regularity properties of $\left(X^{N}\right)$ may thus give an indication of the regularity of the Wasserstein diffusion, although in this paper we deal with the case when the dimension $N$ is fixed. Here our results read as follows. 
Theorem 1.1. For any $\beta>0$, the Dirichlet form $\mathcal{E}^{N}$ generates a strong Feller process $\left(X^{N}\right)$ on $\bar{\Sigma}_{N}$. Moreover, for $\beta \geq(N+1)$ the associated heat kernel $\left(P_{t}\right)$ is exponentially smoothing on Lipschitz functions, i.e. for $t>0$

$$
\operatorname{Lip}\left(P_{t} f\right) \leq \exp \left(-\left(\frac{\beta}{N+1}-1\right) k_{N} \cdot t\right) \operatorname{Lip}(f)
$$

for all $f \in \operatorname{Lip}\left(\bar{\Sigma}_{N}\right)$, where $k_{N}>0$ is a universal constant depending only on $N$.

As a result, the process $X=\left(X_{t}^{x}\right)_{t \geq 0}^{x}$ can be started from any initial configuration $x \in \bar{\Sigma}_{N}$.

A crucial ingredient for the proof of Theorem 1.1 is the following ('Markov-') uniqueness result for the operator $\left(L^{N}, C_{N e u}^{2}\right)$ which is interesting in its own right (cf. [7] for a different approach to a perturbation of independent Bessel-squared processes).

Proposition 1.2. For $\beta<2(N+1)$, there is at most one symmetric strongly continuous contraction semigroup $\left(T_{t}\right)_{t \geq 0}$ on $L^{2}\left(\Sigma_{N}, q_{N}\right)$ whose generator $(\mathcal{L}, \mathcal{D}(\mathcal{L}))$ extends $\left(L^{N}, C_{N e u}^{2}\right)$.

Note that Theorem 1.1 implies that $X$ is a Feller process on $\bar{\Sigma}_{N}$ for all $\beta>0$, i.e. the associated transition semigroup on $L^{2}\left(\Sigma_{N}, q_{N}\right)$ defines a strongly continuous contraction semigroup on the subspace $C\left(\bar{\Sigma}_{N}\right)$ equipped with the sup-norm topology. This leads to the following existence and uniqueness result.

Corollary 1.3. The formal system of Skorokhod SDEs defines via the associated martingale problem a unique $q_{N}$-symmetric diffusion on $\Sigma_{N}$. This process admits a unique extension to allow for arbitrary starting points on the (closed) simplex $\bar{\Sigma}_{N}$.

As for path regularity we obtain the following characterization.

Theorem 1.4. For any starting point $x \in \bar{\Sigma}_{N},\left(X^{x}\right) \in \bar{\Sigma}_{N} \subset \mathbb{R}^{N}$ is a Euclidean semi-martingale if and only if $\beta /(N+1) \geq 1$.

In particular, we obtain that a Skorokhod decomposition of the process $X^{x}$ is impossible if $\beta$ is small enough. This is in sharp contrast to the previous works; cf. [26, 12] (and [5] for the drift-free case). Moreover, again due to the uniqueness assertion of Proposition 1.2 the following negative result holds true.

Corollary 1.5. If $\beta /(N+1)<1$, the system of equations (1.3), (1.4) with initial condition $X_{0} \sim q_{N}$ is ill-posed in the class of $q_{N}$-symmetric diffusions, i.e. it admits no solution in the the sense of Itô calculus.

Theorems 1.1 and 1.4 generalize the corresponding classical results for the family of standard real Bessel processes, which are proved in a completely different manner; cf. [8, 20].

Strategy of proof. While the proof of Theorem 1.4 consists of a straightforward application of a regularity criterion for Dirichlet processes by Fukushima [15], the proof of Theorem 1.1 is more involved, and entirely different methods are used in the two respective cases $\beta<N+1$ and $\beta \geq N+1$. Both cases are non-trivial from an analytic point of view due to the degenerating coefficients and the Neumann boundary condition.

By comparison the case $\beta \geq(N+1)$ is much easier since $q_{N}$ is then log-concave. Using a recent result by Ambrosio, Savaré and Zambotti [2] on the stability of 
gradient flows for the relative entropy functional on the Wasserstein space, the contraction estimate (1.5) is established by smooth approximation and coupling.

For $\beta<(N+1)$ the measure $q_{N}$ is no longer log-concave, and to prove the Feller property we proceed in three main steps. The first crucial observation is that the reference measure $q_{N}$ can be extended to a measure $\hat{q}$ on the full Euclidean space which lies in the Muckenhoupt class $\mathcal{A}_{2}$, allowing for a rich potential theory. In particular, the Poincaré inequality and doubling condition hold which imply via heat kernel estimates the regularity of the induced process on $\mathbb{R}^{N}$. The second step is to use the reflection symmetry of the problem which allows us to treat the Neumann boundary condition indeed via a reflection of a suitable chosen process $Y$ on the flat $N$-dimensional torus. The third step is to identify $X$ with the projection of $Y$ onto $\Sigma_{N}$ by establishing the Markov uniqueness. Here we shall again depend on the nice potential theory available in the Muckenhoupt class.

The partial resemblance of our proof for $\beta<(N+1)$ to the classical work by Bass and Hsu [6] is no surprise. However, we did not find any similar work in the probability literature where potential theory for Muckenhoupt weights was used as extensively as in our case.

\section{Dirichlet FORM AND INTEGRATION BY PARTS FORMUlA}

The rigorous construction of $\left(X^{N}\right)$ departs from the symmetrizing measure $q_{N}$ on $\Sigma_{N}$ defined above in (1.1). Note that one can identify $L^{p}\left(\Sigma_{N}, q_{N}\right)$ with $L^{p}\left(\bar{\Sigma}_{N}, q_{N}\right)$, $p \geq 1$. Throughout the paper $q_{N}$ denotes both the measure and its Lebesque density, i.e. $q_{N}(A)=\int_{A} q_{N}(x) d x$ for all measurable $A \subseteq \bar{\Sigma}_{N}$.

For all $\beta>0, N \in \mathbb{N}$, the measure $q_{N}$ satisfies the 'Hamza condition', because it has a strictly positive density with locally integrable inverse; cf. e.g. 1]. This implies that the form $\mathcal{E}^{N}(f, f)$ with domain $f \in C^{\infty}\left(\bar{\Sigma}_{N}\right)$ is closable on $L^{2}\left(\Sigma_{N}, q_{N}\right)$. The $L^{2}\left(\Sigma_{N}, q_{N}\right)$-closure defines a local regular Dirichlet form, still denoted by $\mathcal{E}^{N}$. General Dirichlet form theory asserts the existence of a Hunt diffusion $\left(X^{N}\right)$ associated with $\mathcal{E}^{N}$ which can be started in $q_{N}$-almost all $x \in \bar{\Sigma}_{N}$ and which is understood as a generalized solution of the systems (1.3), (1.4).

The following integration by parts formula for $q_{N}$ can be easily verified (e.g. by approximation from corresponding integrals over increasing sub-domains $\Sigma_{N}^{\epsilon} \subset$ $\left.\Sigma_{N}\right)$.

Proposition 2.1. Let $u \in C^{1}\left(\bar{\Sigma}_{N}\right)$ and $\xi=\left(\xi^{1}, \ldots, \xi^{N}\right)$ be a vector field in $C^{1}\left(\bar{\Sigma}_{N}, \mathbb{R}^{N}\right)$ satisfying $\langle\xi, \nu\rangle=0$ on $\partial \Sigma_{N}$, where $\nu$ denotes the outward normal field of $\partial \Sigma_{N}$. Then

$$
\begin{aligned}
\int_{\Sigma_{N}} & \langle\nabla u, \xi\rangle q_{N}(d x) \\
& =-\int_{\Sigma_{N}} u\left[\operatorname{div}(\xi)+\left(\frac{\beta}{N+1}-1\right) \sum_{i=1}^{N} \xi^{i}\left(\frac{1}{x^{i}-x^{i-1}}-\frac{1}{x^{i+1}-x^{i}}\right)\right] q_{N}(d x) .
\end{aligned}
$$

Remark 2.2. Let $u \in C^{1}\left(\bar{\Sigma}_{N}\right)$ and $\xi$ be a vector field of the the form $\xi=w \vec{\varphi}$ with $w \in C^{1}\left(\bar{\Sigma}_{N}\right)$ and $\vec{\varphi}(x)=\left(\varphi\left(x^{1}\right), \ldots, \varphi\left(x^{N}\right)\right), \varphi \in C^{\infty}([0,1])$ and $\langle\vec{\varphi}, \nu\rangle=0$ on

$\partial \Sigma_{N}$, in particular $\varphi_{\mid \partial[0,1]}=0$. Then, the integration by parts formula above reads

$$
\int_{\Sigma_{N}}\langle\nabla u, \xi\rangle q_{N}(d x)=-\int_{\Sigma_{N}} u\left[w V_{N, \varphi}^{\beta}+\langle\nabla w, \vec{\varphi}\rangle\right] q_{N}(d x)
$$


where

$$
V_{N, \varphi}^{\beta}\left(x^{1}, \ldots, x^{N}\right):=\left(\frac{\beta}{N+1}-1\right) \sum_{i=0}^{N} \frac{\varphi\left(x^{i+1}\right)-\varphi\left(x^{i}\right)}{x^{i+1}-x^{i}}+\sum_{i=1}^{N} \varphi^{\prime}\left(x^{i}\right) .
$$

Let $C_{N e u}^{2}=\left\{f \in C^{2}\left(\bar{\Sigma}_{N}\right):\langle\nabla f, \nu\rangle=0\right.$ on $\left.\partial \Sigma_{N}\right\}$ as above with $\nu$ still denoting the outer normal field on $\partial \Sigma_{N}$. Then, for any $f \in C^{1}\left(\bar{\Sigma}_{N}\right)$ and $g \in C_{N e u}^{2}$ we apply the integration by parts formula in Proposition 2.1 for $\xi=\nabla g$ to obtain

$$
\mathcal{E}^{N}(f, g)=-\int_{\Sigma_{N}} f L^{N} g q_{N}(d x) .
$$

Moreover, it is easy to show that

$$
\left|\mathcal{E}^{N}(f, g)\right| \leq C\|f\|_{L^{2}\left(\Sigma_{N}, q_{N}\right)}, \quad \forall f \in D\left(\mathcal{E}^{N}\right) .
$$

In particular, $C_{N e u}^{2}$ is contained in the domain of the generator $\mathcal{L}$ associated with $\mathcal{E}^{N}$ and $L^{N} f=\mathcal{L} f$ for all $f \in C_{N e u}^{2}$.

\section{Strong Feller Property}

In the proof of Theorem 1.1 the cases $\beta<(N+1)$ and $\beta \geq(N+1)$ are treated separately. Both cases are not trivial from an analytic perspective due to the combination of degenerate coefficients and the Neumann boundary condition. In fact we could not find any general result in the PDE literature which covers the current model.

3.1. The case $\beta<(N+1)$. For $\beta<(N+1)$ the measure $q_{N}$ is not log-concave. The proof below, however, also extends to all cases when $\beta<2(N+1)$ (cf. Proposition 1.2.).

Let $Q_{N}=\left\{x=\left(x_{1}, \ldots, x_{N}\right) \in \mathbb{R}^{N} \mid\|x\|_{\infty} \leq 1\right\}$ denote the closed $N$-dimensional hypercube of length 2 in $\mathbb{R}^{N}$ and let

$$
T: Q_{N} \rightarrow \bar{\Sigma}_{N}, \quad x \mapsto\left(\left|x^{(1)}\right|, \ldots,\left|x^{(N)}\right|\right),
$$

where $(1), \ldots,(N)$ denotes the permutation of $1, \ldots, N$ such that

$$
\left|x^{(1)}\right| \leq \ldots \leq\left|x^{(N)}\right| \text {. }
$$

To simplify notation the density $q_{N}$ on $\Sigma_{N}$ is extended as a measurable function on $\bar{\Sigma}_{N}$ assuming values in the extended reals by $+\infty$ on $\partial \Sigma_{N}$. Then we define $\hat{q}_{N}: \mathbb{R}^{N} \mapsto \mathbb{R} \cup\{+\infty\}$ by

$$
\hat{q}_{N}(x)=q_{N}(T(x))
$$

Let $\hat{q}_{N}$ also denote the 2-periodic extension on $\mathbb{R}^{N}$ satisfying $\hat{q}_{N}(x+2 k)=\hat{q}_{N}(x)$ for $x, k \in \mathbb{R}^{N}$ and $\|k\|_{\infty}=1$. As above the function $x \mapsto \hat{q}(x)=\hat{q}_{N}(x)$ is identified with the induced $\sigma$-finite measure $\hat{q}_{N}(x) d x$ on $\mathbb{R}^{N}$ and $Q_{N}$, respectively.

Lemma 3.1. For $\beta<2(N+1)$ the measure $\hat{q}$ belongs to the Muckenhoupt class $\mathcal{A}_{2}$, i.e. there exists a positive constant $C(N)$ such that for every Euclidean ball $B_{R}$

$$
\frac{1}{\left|B_{R}\right|} \int_{B_{R}} \hat{q} d x \frac{1}{\left|B_{R}\right|} \int_{B_{R}} \hat{q}^{-1} d x \leq C
$$

where $\left|B_{R}\right|$ denotes the Lebesgue measure of the ball $B_{R}$. 
To prove Lemma 3.1 we will need some more notation. Let $\mathcal{Q}$ be the family of hypercubes defined by

$$
\mathcal{Q}:=\left\{k+Q_{N} \mid k \in(2 \mathbb{Z})^{N}\right\} .
$$

The map $T: Q^{N} \mapsto \Sigma_{N}$ has a natural 2-periodic extension $T: \mathbb{R}^{N} \mapsto \bar{\Sigma}_{N}$ given by $T(x)=T(y)$ if $x=k+y$ with $x \in \mathcal{Q}_{N}$ and $k \in(2 \mathbb{Z})^{N}$. Also, for every $Q \in \mathcal{Q}$,

$$
\int_{Q} \hat{q}_{N}^{ \pm 1} d x=2^{N} N ! \int_{\Sigma_{N}} q_{N}^{ \pm 1} d x
$$

For some small positive $\delta$ set

$$
\Omega_{i}:=\Omega_{i}^{\delta}:=\left\{x \in \bar{\Sigma}_{N}: x^{i+1}-x^{i} \geq \delta\right\}, \quad i=0, \ldots, N .
$$

For any $R>0$ and $m=\left(m_{1}, \ldots, m_{N}\right) \in \mathbb{R}^{N}$ let $P_{R}^{i}(m)$ denote the parallelepiped in $\mathbb{R}^{N}$ given by

$$
\begin{aligned}
P_{R}^{i}(m)= & m+\left\{x \in \mathbb{R}^{N}: x^{1} \in[0, R], x^{2} \in\left[x^{1}, x^{1}+R\right], \ldots, x^{i} \in\left[x^{i-1}, x^{i-1}+R\right],\right. \\
& \left.x^{i+1} \in\left[x^{i+2}-R, x^{i+2}\right], \ldots, x^{N-1} \in\left[x^{N}-R, x^{N}\right], x^{N} \in[1-R, 1]\right\} .
\end{aligned}
$$

Then the following elementary statement holds.

Lemma 3.2. There exist two constants $c_{0}=c_{0}(\delta, N)>0$ and $l=l(N)>0$ such that for all balls $B_{R}$ in $\mathbb{R}^{N}$ with radius $R<c_{0}$ there exists $i \in\{0, \ldots, N\}$ such that $K:=T\left(B_{R}\right) \subset \Omega_{i}^{\delta}$ and $K \subseteq P_{l R}^{i}(m) \subset \Sigma_{N}$ for some $m=\left(m^{1}, \ldots, m^{N}\right) \in Q_{N}$.

Proof of Lemma 3.1. We will consider large and small balls separately.

Case 1. $R>2$. Then $\#\left\{Q \in \mathcal{Q}: B_{R} \cap Q \neq \emptyset\right\} \leq(1+R)^{N}$. Hence, using (3.3) we obtain

$$
\int_{B_{R}} \hat{q}_{N}^{ \pm 1} d x \leq(1+R)^{N} \int_{Q_{N}} \hat{q}_{N}^{ \pm 1} d x=(1+R)^{N} 2^{N} N ! \int_{\Sigma_{N}} q_{N}^{ \pm 1} d x
$$

and since $\left|B_{R}\right| \sim R^{N}$ this gives (3.2).

Case 2. $c_{0} \leq R \leq 2$ with $c_{0}$ as in Lemma 3.2. Similar to the previous case we have $\#\left\{Q \in \mathcal{Q}: B_{R} \cap Q \neq \emptyset\right\} \leq 3^{N}$ so that

$$
\int_{B_{R}} \hat{q}_{N}^{ \pm 1} d x \leq 3^{N} 2^{N} N ! \int_{\Sigma_{N}} q_{N}^{ \pm 1} d x
$$

and since $\left|B_{R}\right| \sim R^{N} \geq c_{0}^{N}$ we again obtain (3.2).

Case 3. $0<R<c_{0}$. In the following the symbol $C$ denotes a positive constant depending on $N$ and $\delta$, possibly changing its value from one occurrence to another. Let $K, i$ and $P_{l R}^{i}(m)$ be as in Lemma 3.2. Then, we observe that by construction

$$
\int_{B_{R}} \hat{q}_{N}^{ \pm 1} d x \leq C \int_{K} q_{N}^{ \pm 1} d x
$$

Moreover, since $K \subset \Omega_{i}^{\delta}$ we have $\delta \leq x^{i+1}-x^{i} \leq 1$ for every $x \in K$, which implies

$$
\begin{aligned}
\int_{K} q_{N}^{ \pm 1} d x & =C \int_{K} \prod_{j=0}^{N}\left(x^{j+1}-x^{j}\right)^{ \pm\left(\frac{\beta}{N+1}-1\right)} d x \leq C \int_{K} \prod_{\substack{0 \leq j \leq N \\
j \neq i}}\left(x^{j+1}-x^{j}\right)^{ \pm\left(\frac{\beta}{N+1}-1\right)} d x \\
& \leq C \int_{P_{l R}^{i}(m)} \prod_{\substack{0 \leq j \leq N \\
j \neq i}}\left(x^{j+1}-x^{j}\right)^{ \pm\left(\frac{\beta}{N+1}-1\right)} d x .
\end{aligned}
$$


By the change of variables $y_{j}=x^{j}-x^{j-1}, j=1, \ldots, i$, and $y_{j}=x^{j+1}-x^{j}$, $j=i+1, \ldots, N$, we obtain

$$
\int_{K} q_{N}^{ \pm 1} d x=\prod_{j=1}^{i} \int_{m_{j}}^{m_{j}+l R} y_{j}^{ \pm\left(\frac{\beta}{N+1}-1\right)} d y_{j} \prod_{j=i+1}^{N} \int_{-m_{j}}^{-m_{j}+l R} y_{j}^{ \pm\left(\frac{\beta}{N+1}-1\right)} d y_{j}
$$

so that

$$
\begin{aligned}
\frac{1}{\left|B_{R}\right|^{2}} & \int_{B_{R}} \hat{q}_{N} d x \int_{B_{R}} \hat{q}_{N}^{-1} d x \\
& \leq C R^{-2 N} \prod_{j=1}^{i} \int_{m_{j}}^{m_{j}+l R} y_{j}^{\frac{\beta}{N+1}-1} d y_{j} \prod_{j=1}^{i} \int_{m_{j}}^{m_{j}+l R} y_{j}^{-\left(\frac{\beta}{N+1}-1\right)} d y_{j} \\
& \times \prod_{j=i+1}^{N} \int_{-m_{j}}^{-m_{j}+l R} y_{j}^{\frac{\beta}{N+1}-1} d y_{j} \prod_{j=i+1}^{N} \int_{-m_{j}}^{-m_{j}+l R} y_{j}^{-\left(\frac{\beta}{N+1}-1\right)} d y_{j} .
\end{aligned}
$$

Recall that we have assumed $\beta<2(N+1)$ and that in one dimension the weight function $x \mapsto|x|^{\eta}$ on $\mathbb{R}$ is contained in $\mathcal{A}_{2}$ if $\eta \in(-1,1)$ (see p. 229 and p. 236 in 25]). Hence, we get for every $j \in\{1, \ldots, N\}$ and $a<b$

$$
\int_{a}^{b} y_{i}^{\frac{\beta}{N+1}-1} d y_{i} \int_{a}^{b} y_{i}^{-\left(\frac{\beta}{N+1}-1\right)} d y_{i} \leq C(b-a)^{2}
$$

and the result follows.

Corollary 3.3 ([27], [1] ). The measure $\hat{q}$ is doubling and satisfies a uniform local Poincaré inequality on $\mathbb{R}^{N}$. That is, there exists a constant $C>0$ such that for all Euclidean balls $B_{R}$,

$$
\hat{q}\left(B_{2 R}\right) \leq C \hat{q}\left(B_{R}\right)
$$

and

$$
\int_{B_{R}}\left|f-(f)_{B_{R}}\right|^{2} d \hat{q} \leq C R^{2} \int_{B_{R}}|\nabla f|^{2} d \hat{q},
$$

and $f \in C^{1}\left(\mathbb{R}^{N}\right)$, where $(f)_{B_{R}}$ denotes the integral $\frac{1}{\hat{q}\left(B_{R}\right)} \int_{B_{R}} f d \hat{q}$.

Let $\mathbb{T}^{N}$ denote the $N$-dimensional torus obtained from $Q_{N}$ by gluing together opposite faces of $\partial Q_{N}$, i.e. $\mathbb{T}^{N}=Q_{N} / \sim$ with

$$
x \sim y \text { for } x, y \in \partial Q_{N} \text { iff }\left|x_{i}-y_{i}\right|= \begin{cases}2 & \text { if } i=j \text { for some } j \in\{1, \ldots, N\}, \\ 0 & \text { else. }\end{cases}
$$

$\mathbb{T}^{N}$ is naturally equipped with the measure $\hat{q}_{\mid Q_{N}}$, which is again denoted by $\hat{q}$, and let $d_{N}: T_{N} \times T_{N} \mapsto \mathbb{R}_{+}$be the induced metric on $\mathbb{T}^{N}$, which coincides locally with the flat Euclidean metric. For $f \in C^{1}\left(\mathbb{T}^{N}\right)$ let

$$
\hat{\mathcal{E}}^{N}(f, f)=\int_{T_{N}}|\nabla f|^{2} \hat{q}(d x)
$$

Then by the same reasons as in section 2 we obtain the closability of $\hat{\mathcal{E}}^{N}$ on $L^{2}\left(\mathbb{T}^{N}, \hat{q}\right)$. Letting $\hat{\mathcal{E}}^{N}$ also denote its closure, thanks to (3.4) and (3.5) the following result is obtained. 
Proposition 3.4. The tuple $\left(\hat{\mathcal{E}}^{N}, D\left(\mathcal{E}^{N}\right)\right)$ is a local Dirichlet space on $L^{2}\left(\mathbb{T}^{N}, \hat{q}\right)$ whose intrinsic metric $d_{\hat{E}^{N}}$ coincides with $d_{N}$. Moreover, $\hat{q}$ is doubling on $\mathbb{T}^{N}$ and $\hat{\mathcal{E}}^{N}$ supports a local Poincaré inequality in the sense of conditions Ib) and Ic) of 23.

Corollary 3.5 ([23]). Let $Y$ be the $\hat{q}$-symmetric diffusion on $\mathbb{T}^{N}$ associated to $\hat{E}^{N}$ on $L^{2}\left(\mathbb{T}^{N}, \hat{q}\right)$. Then $Y$ admits a symmetric $d_{N}$-Hölder-continuous heat kernel on $\mathbb{T}^{N}$. In particular, $Y$ is a strong Feller process.

Note for the map $T: Q_{N} \mapsto \Sigma_{N}$ that $T(x)=T(y)$ for boundary points $x, y \in$ $\partial Q_{N}$ with $x \sim y$. Hence the map is also well defined and continuous from $\mathbb{T}^{N}$ to $\bar{\Sigma}_{N}$. Moreover, $T$ is a local isometry.

Lemma 3.6. Define the process $\tilde{X}$. $=T(Y.) \in \bar{\Sigma}_{N}$. Then $\tilde{X}$ is Markovian and $q_{N}$-symmetric. Moreover, $\tilde{X}$ admits a (symmetric) Hölder-continuous transition kernel. In particular, $\tilde{X}$ is a strong Feller process on $\bar{\Sigma}_{N}$.

Proof. The Markov property is implied e.g. by the condition that for any Borel set $A \subseteq \bar{\Sigma}_{N}$

$$
\mathbb{P}_{x}\left[Y_{t} \in T^{-1}(A)\right]=\mathbb{P}_{y}\left[Y_{t} \in T^{-1}(A)\right] \quad \text { whenever } T(x)=T(y) .
$$

Now the choice of $\hat{q}$ implies that for any Borel set $A \subseteq \bar{\Sigma}_{N}$ condition (3.7) is satisfied. To see this, let $\left\{\sigma_{k} \mid k=1, \ldots, N+N(N-1) / 2\right\}$ be the collection of linereflections in $\mathbb{R}^{N}$ with respect to either one of the coordinate axes $\left\{\lambda e_{i}, \lambda \in \mathbb{R}\right\}$ or a diagonal $\left\{\lambda\left(e_{j}+e_{k}\right)\right\}$. Then for $x, y \in \mathbb{T}^{N}$ with $T(x)=T(y)$ there exists a finite sequence $\sigma_{k_{1}}, \ldots, \sigma_{k_{l}}$ such that $\tau(x):=\sigma_{k_{1}} \circ \sigma_{k_{2}} \circ \ldots \circ \sigma_{k_{l}}(x)=y$. Now each of the reflections $\sigma_{i}$ preserves the Dirichlet form (3.6) such that the processes $\tau\left(Y^{x}\right)$ and $Y^{y}$ are equal in distribution. Moreover, $\tau\left(T^{-1}(A)\right)=T^{-1}(A)$, from which (3.7) is obtained. The $\hat{q}$-symmetry of $Y$ implies the $q_{N}$-symmetry of $\tilde{X}$ because $\hat{q}$ is the image measure of $q_{N}$ under $T$. The strong Feller property of $\tilde{X}$ is immediate from the fact that $Y$ is strong Feller and that $T$ is continuous and locally invertible with continuous inverse.

In order to complete the proof of Theorem 1.1, it remains to identify the laws of the processes $\tilde{X}$ and $X$. This will be achieved by showing that both $\tilde{X}$ and $X$ solve the same martingale problem on a large enough set of test functions. To this aim note that as in section 2 the following integration by parts formula holds for the generator of $\hat{\mathcal{L}}$ the Dirichlet form $\hat{\mathcal{E}}^{N}$ :

$$
\hat{\mathcal{E}}^{N}(f, g)=-\int_{\mathbb{T}^{N}} g(x) \hat{L}_{N} f(x) \hat{q}(d x) \quad \forall g \in C^{1}\left(\mathbb{T}^{N}\right), f \in C_{N e u(\mathscr{S})}^{2}\left(\mathbb{T}^{N}\right),
$$

where $\mathscr{S}=\left\{x \in \mathbb{T}^{N} \mid \hat{q}(x)=\infty\right\}$,

$C_{\text {Neu }(\mathscr{S})}^{2}\left(\mathbb{T}^{N}\right)=\left\{f \in C^{2}\left(\mathbb{T}^{N}\right) \mid \nabla f \cdot \nu_{\mathscr{S}}=0\right.$ on all $(n-1)$-dimensional faces of $\left.\mathscr{S}\right\}$

with $\nu_{\mathscr{S}}$ denoting (any choice of) the normal vector to the subset $\mathscr{S} \subset \mathbb{T}^{N}$, and for $x \in \mathbb{T}^{N} \backslash \mathscr{S}$

$$
\hat{L}^{N} f(x)=\left(\frac{\beta}{N+1}-1\right) \sum_{i=1}^{N}\left(\frac{1}{x^{(i)}-x^{(i-1)}}-\frac{1}{x^{(i+1)}-x^{(i)}}\right) \frac{\partial}{\partial x^{(i)}} f(x)+\Delta f(x) .
$$

Recall that in the formula above $(1), \ldots,(N)$ denotes the permutation of $1, \ldots, N$ such that $\left|x^{(1)}\right| \leq \ldots \leq\left|x^{(N)}\right|$. In particular, $C_{N e u(\mathscr{S})}^{2}\left(\mathbb{T}^{N}\right)$ is contained in the 
domain of $\hat{\mathcal{L}}$. Finally, since $f \circ T \in C_{N e u(\mathscr{S})}^{2}\left(\mathbb{T}^{N}\right)$ for $f \in C_{\text {Neu }}^{2}(\bar{\Sigma}), \hat{L}^{N}(f \circ T)=$ $\left(L^{N} f\right) \circ T$ in this case and the measure $\hat{q}$ is preserved (up to the factor $2^{N} \cdot N$ !) by the map $T: \mathbb{T}^{N} \mapsto \bar{\Sigma}_{N}$, the following assertion holds.

Lemma 3.7. The process $\tilde{X}$ solves the martingale problem for $\left(L_{N}, C_{N e u}^{2}\left(\bar{\Sigma}_{N}\right)\right)$ on $\bar{\Sigma}_{N}$ with initial condition $q_{N} \in \mathcal{M}_{1}\left(\bar{\Sigma}_{N}\right)$.

Since both $\tilde{X}$ and $X$ are Markovian, $q_{N}$-symmetric solutions to the martingale problem for $\left(L_{N}, C_{N e u}^{2}\left(\bar{\Sigma}_{N}\right)\right)$, it suffices to show that there is at most one Markov semigroup on $L^{2}\left(\Sigma_{N}, q_{N}\right)$ with these properties. This is the Markov uniqueness property of $\left(L^{N}, C_{N e u}^{2}\left(\Sigma_{N}\right), q_{N}\right)$ as stated in Proposition 1.2 cf. 10, Chap. 1., Definition 1.2].

Proof of Proposition 1.2. Let $H^{1,2}\left(\Sigma_{N}, q_{N}\right)$ (resp. ' $H_{0}^{1,2}\left(\Sigma_{N}, q_{N}\right)$ ' in the notation of [10]) denote the closure of $C_{N e u}^{2}$ w.r.t. to the norm

$$
\|f\|_{1}=\left(\|f\|_{L^{2}\left(\Sigma_{N}, q_{N}\right)}^{2}+\|\nabla f\|_{L^{2}\left(\Sigma_{N}, q_{N}\right)}^{2}\right)^{1 / 2}
$$

and let $W^{1,2}$ be the Hilbert space of $L^{2}\left(\Sigma_{N}, q_{N}\right)$-functions $f$ whose distributional derivative $D f$ is in $L^{2}\left(\Sigma_{N}, q_{N}\right)$, equipped with the norm

$$
\|f\|_{1}=\left(\|f\|_{L^{2}\left(\Sigma_{N}, q_{N}\right)}^{2}+\|D f\|_{L^{2}\left(\Sigma_{N}, q_{N}\right)}^{2}\right)^{1 / 2} .
$$

Clearly, the quadratic form $Q(f, f)=\langle D f, D f\rangle_{L^{2}\left(\Sigma_{N}, q_{N}\right)}, \mathcal{D}(Q)=W^{1,2}\left(\Sigma_{N}, q_{N}\right)$, is a Dirichlet form on $L^{2}\left(\Sigma_{N}, q_{N}\right)$. Hence we may use the basic criterion for Markov uniqueness [10, Chap. 3, Corollary 3.2], according to which $\left(L, C_{N e u}^{2}\right)$ is Markov unique if $H^{1,2}=W^{1,2}$.

To prove the latter it obviously suffices to prove that $H^{1,2}$ is dense in $W^{1,2}$. For any $f \in W^{1,2}\left(\Sigma_{N}, q_{N}\right)$ we first show that $f$ can be approximated w.r.t. $\|\cdot\|_{1}$ by functions which are smooth up to boundary and secondly that such functions can be approximated in $\|\cdot\|_{1}$ by smooth Neumann functions.

Due to Lemma 3.1 the extension $\hat{q}(x)=\hat{q}(T(x)), x \in \mathbb{R}^{N}$, lies in the Muckenhoupt class $\mathcal{A}_{2}$. Further note that $W^{1,2}\left(\Sigma_{N}, \hat{q}\right) \subset W^{1,2}\left(\Sigma_{N}^{\delta}, d x\right)=H^{1,2}\left(\Sigma_{N}^{\delta}, d x\right)$ if $\beta \leq N+1$, and $W^{1,2}\left(\Sigma_{N}^{\delta}, \hat{q}\right) \subset W^{1,1}\left(\Sigma_{N}^{\delta}, d x\right)=H^{1,1}\left(\Sigma_{N}^{\delta}, d x\right)$ by the Hölder inequality if $N+1<\beta<2(N+1)$, such that $f$ has well defined boundary values in $L^{1}\left(\partial \Sigma_{N}, d x\right)$. Hence we may conclude that the extension $\hat{f}(x)=f(T(x)), x \in$ $[-1,3]^{N}$, can again be extended to a weakly differentiable function $\hat{f} \in W^{1,2}\left(\mathbb{R}^{N}, \hat{q}\right)$. By [17, Theorem 2.5] the mollification with the standard mollifier yields an approximating sequence $\left\{u_{l}\right\}_{l}$ of smooth functions $u_{l} \in C_{0}^{\infty}\left(\mathbb{R}^{N}\right)$ of $\hat{f}$ in the weighted Sobolev spaces $H^{1,2}\left(\mathbb{R}^{N}, \hat{q}\right)$. Hence, we obtain a sequence of $C^{\infty}\left(\bar{\Sigma}_{N}\right)$-functions $\left\{u_{l}\right\}_{l}$ which converges to $f$ in $H^{1,2}\left(\Sigma_{N}, q_{N}\right)$. This finishes the first step.

In the second step we thus may assume w.l.o.g. that $f$ is smooth up to the boundary of $\Sigma_{N}$. In particular, $f$ is globally Lipschitz. Since $q_{N}$ is integrable on $\Sigma_{N}$ we may modify $f$ close to the boundary to obtain a Lipschitz function $\tilde{f}$ which satisfies the Neumann condition and which is close to $f$ in $\|\cdot\|_{1}$. (Take, e.g. $\tilde{f}(x)=f(\pi(x))$, where $\pi(x)$ is the projection of $x$ into the set $\Sigma_{N}^{\epsilon}=\{x \in$ $\left.\Sigma_{N}, \mid \operatorname{dist}\left(x, \partial \Sigma_{N}\right) \geq \epsilon\right\}$ for small $\epsilon>0$. W We may now proceed as in step one to obtain an approximation of $\tilde{f}$ by smooth functions w.r.t. $\|.\|_{1}$, where we note that neither extension by reflection through the map $T$ nor the standard mollification in [17] of the extended $\tilde{f}$ destroys the Neuman boundary condition. 
Hence we arrive at the following statement which implies the first statement of Theorem 1.1 in the case of $\beta<2(N+1)$.

Corollary 3.8. For quasi-every $x \in \bar{\Sigma}_{N}$, the processes $\tilde{X}^{x}$ and $X^{x}$ are equal in law. In particular, $X$ is a strong Feller process on $\bar{\Sigma}_{N}$.

3.2. The case $\beta \geq(N+1)$. The estimate (1.5) looks like a straightforward application of the Bakry-Emery $\Gamma_{2}$-calculus. However, the complete Bakry-Emery criterion requires the $\Gamma_{2}$-condition on an algebra of functions which is dense in the domain of the generator $\mathcal{L}$ of $\mathcal{E}$ w.r.t. the graph norm. The verification of the latter typically leads back to elliptic regularity theory for $\mathcal{L}$, which we want to avoid. Instead our argument below is based on a recent powerful result by Ambrosio, Savare and Zambotti [2] on the stability of reversible processes with log-concave invariant measures.

Proof of 1.5 . By abuse of notation let $q_{N}$ be the extension of the measure $q_{N}$ to $\mathbb{R}^{N}$ given by

$$
q_{N}(A)=q_{N}\left(A \cap \Sigma_{N}\right)=\frac{1}{Z_{\beta}} \int_{A \cap\{V<\infty\}} e^{-\left(\frac{\beta}{N+1}-1\right) V(x)} d x
$$

for any Borel set $A \subset \mathbb{R}^{N}$, where $V: \mathbb{R}^{N} \rightarrow \mathbb{R} \cup\{\infty\}$ and

$$
V(x)= \begin{cases}-\sum_{i=1}^{N+1} \ln \left(x_{i}-x_{i-1}\right), & x \in \Sigma_{N}, \\ \infty, & x \in \mathbb{R}^{N} \backslash \Sigma_{N},\end{cases}
$$

is a lower semicontinuous and convex function on $\mathbb{R}^{N}$. In fact, by elementary calculations, for $x \in \Sigma_{N}$ with $\rho_{i}=\rho_{i}(x)=1 /\left(x_{i}-x_{i-1}\right)^{2}, i=1, \ldots, N+1$,

$$
\left\langle\xi, \nabla^{2} V(x) \xi\right\rangle=\rho_{1} \xi_{1}^{2}+\sum_{i=1}^{N-1} \rho_{i+1}\left(\xi_{i+1}-\xi_{i}\right)^{2}+\rho_{N+1} \xi_{N}^{2} \geq k_{N}|\xi|^{2}, \quad \forall \xi \in \mathbb{R}^{N}
$$

where $k_{N}>0$ denotes the smallest eigenvalue of the strictly elliptic matrix $A=$ $\left(2 \delta_{i j}-\delta_{1,|i-j|}\right) \in \mathbb{R}^{\mathbb{N} \times \mathbb{N}}$. Hence the measure $q_{N}$ is log-concave in the sense of [2]. (Note that according to ibid. Theorem 1.2.b the Feller property of $X$. on $\bar{\Sigma}_{N}$ is automatically implied.) Let $\bar{x} \in \Sigma_{N}$ denote the barycenter of the simplex $\Sigma_{N}$. For $\epsilon>0$ let

$$
V_{\epsilon}(x)= \begin{cases}V((1-\epsilon) x+\epsilon \bar{x})), & x \in \bar{\Sigma}_{N} \\ \infty, & x \in \mathbb{R}^{N} \backslash \bar{\Sigma}_{N},\end{cases}
$$

and define the measure $q_{N}^{\epsilon}$ on $\mathbb{R}^{N}$ by

$$
q_{N}^{\epsilon}(A)=\frac{1}{Z_{\beta}} \int_{A \cap \Sigma_{N}} e^{-\left(\frac{\beta}{N+1}-1\right) V_{\epsilon}(x)} d x .
$$

Since $V_{\epsilon} \in C^{\infty}\left(\Sigma_{N}\right)$ and the boundary of $\Sigma_{N}$ is piecewise smooth, the corresponding reflecting process can also be constructed by the associated Skorokhod SDE. Moreover, the classical coupling by reflection method can be applied; cf. 29]. Taking expectations in [29, eq. (2.5)] (note that $I=0$ in our case) and using the strict convexity of $V_{\epsilon}$ together with Gronwall's lemma yields the estimate

$$
\mathbb{E}_{C}\left(\bar{X}_{t}^{\epsilon, 1}, \bar{X}_{t}^{\epsilon, 2}\right) \leq e^{-\left(\frac{\beta}{N+1}-1\right)(1-\epsilon)^{2} k_{N} t} d(x, y),
$$

where $C$ denotes the law of the coupling process $\left(\bar{X}_{t}^{\epsilon, 1}, \bar{X}_{t}^{\epsilon, 2}\right)_{t \geq 0}$, starting in $(x, y)$. In particular the following estimate in the $L^{1}$-Wasserstein distance $d_{1}$ for the heat 
kernel of $\left(X^{\epsilon}\right)$ is obtained:

$$
d_{1}\left(P_{t}^{\epsilon}(x, \cdot), P_{t}^{\epsilon}(y, \cdot)\right) \leq e^{-\left(\frac{\beta}{N+1}-1\right)(1-\epsilon)^{2} k_{N} t} d(x, y) .
$$

Since $q_{N}^{\epsilon} \rightarrow q_{N}$ weakly, we may now invoke Theorem 6.1 of 2 in order to pass to the limit for $\epsilon \rightarrow 0$ on the left hand side above, also using the continuity of the $L^{1}$ w.r.t. the $L^{2}$-Wassserstein metric. Hence we arrive at

$$
d_{1}\left(P_{t}(x, \cdot), P_{t}(y, \cdot)\right) \leq e^{-\left(\frac{\beta}{N+1}-1\right)(1-\epsilon)^{2} k_{N} t} d(x, y)
$$

for all $\epsilon>0$ small enough, thus also for $\epsilon=0$. Via Kantorovich duality this implies

$$
\left|P_{t} f(x)-P_{t} f(y)\right| \leq e^{-\left(\frac{\beta}{N+1}-1\right) k_{N} t} \operatorname{Lip}(f) d(x, y)
$$

for all $f \in \operatorname{Lip}\left(\Sigma_{N}\right)$ and $x, y \in \Sigma_{N}$, which is the claim.

\section{Semi-martingale Properties}

In this final section we prove the semi-martingale properties of $X$ stated in Theorem 1.4. To that aim we establish the semi-martingale properties for the symmetric process $X^{N}$ started in equilibrium, which imply the semi-martingale properties to hold for quasi-every starting point $x \in \bar{\Sigma}_{N}$ and by the Feller properties proven in the last section for every starting point $x \in \bar{\Sigma}_{N}$. In order to establish the semi-martingale properties of the stationary process, we shall use the following criterion established by Fukushima in [15. For every open set $G \subset \bar{\Sigma}_{N}$ we set

$$
\mathcal{C}_{G}:=\left\{u \in D\left(\mathcal{E}^{N}\right) \cap C\left(\bar{\Sigma}_{N}\right): \operatorname{supp}(u) \subset G\right\} .
$$

Theorem 4.1. For $u \in D\left(\mathcal{E}^{N}\right)$ the additive functional $u\left(X_{t}^{N}\right)-u\left(X_{0}^{N}\right)$ is a semimartingale if and only if one of the following (equivalent) conditions holds:

i) For any relatively compact open set $G \subset \bar{\Sigma}_{N}$, there is a positive constant $C_{G}$ such that

$$
\left|\mathcal{E}^{N}(u, v)\right| \leq C_{G}\|v\|_{\infty}, \quad \forall v \in \mathcal{C}_{G} .
$$

ii) There exists a signed Radon measure $\nu$ on $\bar{\Sigma}_{N}$ charging no set of zero capacity such that

$$
\mathcal{E}^{N}(u, v)=-\int_{\bar{\Sigma}_{N}} v(x) \nu(d x), \quad \forall v \in C\left(\bar{\Sigma}_{N}\right) \cap \mathcal{D}\left(\mathcal{E}^{N}\right) .
$$

Proof. See Theorem 6.3 in [15].

Theorem 4.2. Let $X^{N}$ be a symmetric diffusion on $\bar{\Sigma}_{N}$ associated with the Dirichlet form $\mathcal{E}^{N}$. Then $X^{N}$ is an $\mathbb{R}^{N}$-valued semi-martingale if and only if $\beta /(N+1) \geq 1$.

Proof. Since the semi-martingale property for $\mathbb{R}^{N}$-valued diffusions is defined componentwise, we shall apply Fukushima's criterion for $u(x)=x^{i}, i=1, \ldots, N$. 
Let us first consider the case where $\beta^{\prime}:=\beta /(N+1)>1$. Then, for a relatively compact open set $G \subset \bar{\Sigma}_{N}$ and $v \in \mathcal{C}_{G}$,

$$
\begin{aligned}
\mathcal{E}^{N}(u, v)= & \int_{\Sigma_{N}} \frac{\partial}{\partial x^{i}} v(x) q_{N}(d x) \\
= & \frac{1}{Z_{\beta}} \int_{0}^{1} d x^{1} \int_{x^{1}}^{1} d x^{2} \ldots \int_{x^{i-1}}^{1} d x^{i+1} \int_{x^{i+1}}^{1} d x^{i+2} \\
& \cdots \int_{x^{N-1}}^{1} d x^{N} \prod_{\substack{j=0 \\
j \neq i-1, i}}^{N}\left(x^{j+1}-x^{j}\right)^{\beta^{\prime}-1} \\
& \times \int_{x^{i-1}}^{x^{i+1}} \frac{\partial}{\partial x^{i}} v(x)\left(x^{i}-x^{i-1}\right)^{\beta^{\prime}-1}\left(x^{i+1}-x^{i}\right)^{\beta^{\prime}-1} d x^{i} .
\end{aligned}
$$

Since $\beta^{\prime}>1$, we obtain by integration by parts,

$$
\begin{aligned}
\int_{x^{i-1}}^{x^{i+1}} \frac{\partial}{\partial x^{i}} v(x)\left(x^{i}-x^{i-1}\right)^{\beta^{\prime}-1}\left(x^{i+1}-x^{i}\right)^{\beta^{\prime}-1} d x^{i} & \\
=-\left(\beta^{\prime}-1\right) \int_{x^{i-1}}^{x^{i+1}} v(x)[ & \left(x^{i}-x^{i-1}\right)^{\beta^{\prime}-2}\left(x^{i+1}-x^{i}\right)^{\beta^{\prime}-1} \\
& \left.-\left(x^{i}-x^{i-1}\right)^{\beta^{\prime}-1}\left(x^{i+1}-x^{i}\right)^{\beta^{\prime}-2}\right] d x^{i},
\end{aligned}
$$

so that

$$
\begin{aligned}
& \left|\int_{x^{i-1}}^{x^{i+1}} \frac{\partial}{\partial x^{i}} v(x)\left(x^{i}-x^{i-1}\right)^{\beta^{\prime}-1}\left(x^{i+1}-x^{i}\right)^{\beta^{\prime}-1} d x^{i}\right| \\
\leq & \left(\beta^{\prime}-1\right)\|v\|_{\infty}\left(\int_{x^{i-1}}^{x^{i+1}}\left(x^{i}-x^{i-1}\right)^{\beta^{\prime}-2} d x^{i}+\int_{x^{i-1}}^{x^{i+1}}\left(x^{i+1}-x^{i}\right)^{\beta^{\prime}-2} d x^{i}\right) \\
\leq & 2\left(\beta^{\prime}-1\right)\|v\|_{\infty} \int_{0}^{1} r^{\beta^{\prime}-2} d r \leq C\|v\|_{\infty},
\end{aligned}
$$

and we obtain that condition (4.1) holds. For $\beta^{\prime}=1$ the measure $q_{N}$ coincides with the normalized Lebesgue measure on $\bar{\Sigma}_{N}$; condition (4.1) follows directly.

Now let $\beta^{\prime}<1$ and let us assume that $u\left(X_{t}^{N}\right)-u\left(X_{0}^{N}\right)$ is a semi-martingale. Then, there exists a signed Radon measure $\nu$ on $\bar{\Sigma}_{N}$ satisfying (4.2). Let $\nu=\nu_{1}-\nu_{2}$ be the Jordan decomposition of $\nu$, i.e. $\nu_{1}$ and $\nu_{2}$ are positive Radon measures. By the previous calculations we have for each relatively compact open set $G \subset \bar{\Sigma}_{N}$ and for all $v \in \mathcal{C}_{G}$,

$$
\begin{aligned}
\mathcal{E}^{N}(u, v)= & -\frac{1}{Z_{\beta}}\left(\beta^{\prime}-1\right) \int_{G} v(x) \prod_{\substack{j=0 \\
j \neq i-1, i}}^{N}\left(x^{j+1}-x^{j}\right)^{\beta^{\prime}-1} \\
& \times\left[\left(x^{i}-x^{i-1}\right)^{\beta^{\prime}-2}\left(x^{i+1}-x^{i}\right)^{\beta^{\prime}-1}\right. \\
& \left.\quad-\left(x^{i}-x^{i-1}\right)^{\beta^{\prime}-1}\left(x^{i+1}-x^{i}\right)^{\beta^{\prime}-2}\right] d x .
\end{aligned}
$$


Hence, we obtain for the Jordan decomposition $\nu=\nu_{1}-\nu_{2}$ that

$$
\begin{aligned}
& \nu_{1}(G)=\frac{1}{Z_{\beta}}\left(1-\beta^{\prime}\right) \int_{G}\left(x^{i+1}-x^{i}\right)^{\beta^{\prime}-2} \prod_{\substack{j=0 \\
j \neq i}}^{N}\left(x^{j+1}-x^{j}\right)^{\beta^{\prime}-1} d x, \\
& \nu_{2}(G)=\frac{1}{Z_{\beta}}\left(1-\beta^{\prime}\right) \int_{G}\left(x^{i}-x^{i-1}\right)^{\beta^{\prime}-2} \prod_{\substack{j=0 \\
j \neq i-1}}^{N}\left(x^{j+1}-x^{j}\right)^{\beta^{\prime}-1} d x .
\end{aligned}
$$

Set $\partial \Sigma_{N}^{j}:=\left\{x \in \partial \Sigma_{N}: x^{j}=x^{j+1}\right\}, j=0, \ldots, N$, and let for some $x_{0} \in \partial \Sigma^{i}$ and $r>0$ that $A:=x_{0}+[-r, r]^{N} \cap \bar{\Sigma}_{N}$ be such that $\operatorname{dist}\left(A, \partial \Sigma_{N}^{j}\right)>0$ for all $j \neq i$. Furthermore, let $\left(A_{n}\right)_{n}$ be a sequence of compact subsets of $A$ such that $A_{n} \uparrow A$ and $\operatorname{dist}\left(A_{n}, \partial \Sigma_{N}^{i}\right)>0$ for every $n$.

By the inner regularity of the Radon measures $\nu_{1}$ and $\nu_{2}$ we have $v_{1}(A)=$ $\lim _{n} \nu_{1}\left(A_{n}\right)$ and $v_{2}(A)=\lim _{n} \nu_{2}\left(A_{n}\right)$. Since $\beta^{\prime}-2<-1$, we get by the choice of $A$ that $\nu_{1}(A)=\infty$, while $\nu_{2}(A)<\infty$, which contradicts the local finiteness of $\nu$ and $\nu_{1}$, respectively.

\section{REFERENCES}

[1] S. Albeverio and M. Röckner. Classical Dirichlet forms on topological vector spacesclosability and a Cameron-Martin formula. J. Funct. Anal., 88(2):395-436, 1990. MR:1038449 (91g:47030)

[2] L. Ambrosio, G. Savaré, and L. Zambotti. Existence and stability for Fokker-Planck equations with log-concave reference measure. Probab. Theory Related Fields, 145(3-4):517-564, 2009. MR2529438 (2010k:60271)

[3] S. Andres and M.-K. von Renesse. Particle approximation of the Wasserstein diffusion. J. Funct. Anal., 258(11):3879-3905, 2010. MR2606878 (2011b:60224)

[4] R. F. Bass, K. Burdzy, and Z.-Q. Chen. Uniqueness for reflecting Brownian motion in lip domains. Ann. Inst. H. Poincaré Probab. Statist., 41(2):197-235, 2005. MR2124641 (2005k:60174)

[5] R. F. Bass and P. Hsu. The semimartingale structure of reflecting Brownian motion. Proc. Amer. Math. Soc., 108(4):1007-1010, 1990. MR.1007487(91a:60205)

[6] R. F. Bass and P. Hsu. Some potential theory for reflecting Brownian motion in Hölder and Lipschitz domains. Ann. Probab., 19(2):486-508, 1991. MR1106272 (92i:60142)

[7] R. F. Bass and E. A. Perkins. Degenerate stochastic differential equations with Hölder continuous coefficients and super-Markov chains. Trans. Amer. Math. Soc., 355(1):373-405 (electronic), 2003. MR 1928092 (2003m:60144)

[8] J. Bertoin. Excursions of a $\operatorname{BES}_{0}(d)$ and its drift term $(0<d<1)$. Probab. Theory Related Fields, 84(2):231-250, 1990. MR.1030728(91e:60229)

[9] E. Cépa and D. Lépingle. Diffusing particles with electrostatic repulsion. Probab. Theory Related Fields, 107(4):429-449, 1997. MR1440140(98k:60177)

[10] A. Eberle. Uniqueness and non-uniqueness of semigroups generated by singular diffusion operators. Springer, 1999. MR.1734956 (2001c:60122)

[11] E. B. Fabes, C. E. Kenig, and R. P. Serapioni. The local regularity of solutions of degenerate elliptic equations. Comm. Partial Differential Equations, 7(1):77-116, 1982. MR643158 (84i:35070)

[12] T. Fattler and M. Grothaus. Strong Feller properties for distorted Brownian motion with reflecting boundary condition and an application to continuous $N$-particle systems with singular interactions. J. Funct. Anal., 246(2):217-241, 2007. MR2321042 (2008b:60178)

[13] J. Fritz and R. L. Dobrushin. Non-equilibrium dynamics of two-dimensional infinite particle systems with a singular interaction. Comm. Math. Phys., 65(1):96, 1979. MR.1552623

[14] M. Fukushima. A construction of reflecting barrier Brownian motions for bounded domains. Osaka J. Math., 4:183-215, 1967. MR0231444(37:6999) 
[15] M. Fukushima. On semi-martingale characterizations of functionals of symmetric Markov processes. Electron. J. Probab., 4:18, 32 pp., 1999. MR.1741537(2001b:60091)

[16] D. J. Grabiner. Brownian motion in a Weyl chamber, non-colliding particles, and random matrices. Ann. Inst. H. Poincaré Probab. Statist., 35(2):177-204, 1999. MR 1678525 (2000i:60091)

[17] T. Kilpeläinen. Weighted Sobolev spaces and capacity. Ann. Acad. Sci. Fenn. Ser. A I Math., 19(1):95-113, 1994. MR1246890 (95h:46054)

[18] P.-L. Lions and A.-S. Sznitman. Stochastic differential equations with reflecting boundary conditions. Comm. Pure Appl. Math., 37(4):511-537, 1984. MR/745330 (85m:60105)

[19] H. Osada. Dirichlet form approach to infinite-dimensional Wiener processes with singular interactions. Comm. Math. Phys., 176(1):117-131, 1996. MR.1372820 (96m:60178)

[20] D. Revuz and M. Yor. Continuous martingales and Brownian motion. Springer, third edition, 1999. MR1725357(2000h:60050)

[21] H. Spohn. Interacting Brownian particles: A study of Dyson's model. In Hydrodynamic behavior and interacting particle systems (Minneapolis, Minn., 1986), pages 151-179. Springer, New York, 1987. MR914993 (89k:82016)

$[22]$ D. W. Stroock. Diffusion semigroups corresponding to uniformly elliptic divergence form operators. In Séminaire de Probabilités, XXII. Springer, Berlin, 1988. MR960535|(90b:35071)

[23] K. T. Sturm. Analysis on local Dirichlet spaces. III. The parabolic Harnack inequality. J. Math. Pures Appl. (9), 75(3):273-297, 1996. MR1387522(97k:31010)

[24] H. Tanaka. Stochastic differential equations with reflecting boundary condition in convex regions. Hiroshima Math. J., 9(1):163-177, 1979. MR529332 (80k:60075)

[25] A. Torchinsky. Real-variable methods in harmonic analysis. Academic Press Inc., 1986. MR869816 (88e:42001)

[26] G. Trutnau. Skorokhod decomposition of reflected diffusions on bounded Lipschitz domains with singular non-reflection part. Probab. Theory Related Fields, 127(4):455-495, 2003. MR2021192 (2004j:60173)

[27] B. O. Turesson. Nonlinear potential theory and weighted Sobolev spaces. Springer, 2000. MR1774162 (2002f:31027)

[28] M.-K. von Renesse and K.-T. Sturm. Entropic measure and Wasserstein diffusion. Ann. Probab., 37(3):1114-1191, 2009. MR2537551 (2010k:60185)

[29] F. Y. Wang. Application of coupling methods to the Neumann eigenvalue problem. Probab. Theory Related Fields, 98(3):299-306, 1994. MR1262968 (94k:58153)

Department of Mathematics, Technische Universität Berlin, Strasse des 17, Juni 136, 10623 Berlin, Germany

E-mail address: andres@math.tu-berlin.de

Department of Mathematics, Technische Universität Berlin, Strasse des 17, Juni 136, 10623 Berlin, Germany

E-mail address: mrenesse@math.tu-berlin.de 